\title{
Some Exact and Explicit Solutions for Nonlinear Schrödinger Equations
}

\author{
İ. ASLAN* \\ Department of Mathematics, Izmir Institute of Technology, Urla, Izmir 35430, Turkey \\ (Received May 28, 2012; in final form October 22, 2012)
}

\begin{abstract}
Nonlinear models occur in many areas of applied physical sciences. This paper presents the first integral method to carry out the integration of Schrödinger-type equations in terms of traveling wave solutions. Through the established first integrals, exact traveling wave solutions are obtained under some parameter conditions.
\end{abstract}

DOI: $10.12693 /$ APhysPolA.123.16

PACS: 02.30.Jr, 02.30.Hq, 04.20.Jb

\section{Introduction}

The physical models that evolve over time, commonly referred to as evolution equations, have been a crucial component of the mathematical description of complex phenomena. Nonlinear evolution equations (NEEs) arise in a vast assortment of fields, ranging from the physical sciences including thermodynamics, soil mechanics, civil engineering, and non-Newtonian fluids to the natural sciences including population ecology, infectious disease epidemiology, and neural networks, etc. Thus, throughout the past few decades, a particular attention has been given to the problem of finding exact solutions of NEEs. By virtue of these solutions, one may give better insight into the physical aspects of the nonlinear models studied. Among the others, certain special form solutions of NEEs may depend only on a single combination of the so-called traveling wave variables because such equations are often described by wave phenomena.

Not all equations are solvable. To carry out the integration of NEEs in terms of analytic solutions, a considerable number of analytic methods have been successfully established and developed on this direction; just to mention a few, the Painleve expansion method [1], inverse scattering method [2], Hirota's bilinear method [3], transformed rational function method [4], symmetry method [5], tanh function method [6], homogeneous balance method [7], F-expansion method [8], (G'/G)-expansion method [9], exp-function method [10], homotopy perturbation method [11], the solitary wave ansatz method [12], further improved F-expansion method [13], multiple exp-function method [14], Adomian-Pade technique [15], etc.

More lately, the superposition principle was successfully used to find exponential traveling wave solutions to the Hirota bilinear equations [16]. But, it is usually hard and time consuming to tackle various kinds of nonlinear problems via the well-known traditional approaches because they are usually restricted and cannot be im-

*e-mail: ismailaslan@iyte.edu.tr plemented to numerous realistic mathematical/physical scenarios.

Recently, by means of the ring theory of commutative algebra, Feng et al. [17-19] developed an effective method which is currently recognized as the first integral method (or the algebraic curve method) to analyze NEEs in terms of exact and explicit solutions. The method has been shown to be a useful tool for solving some nonlinear problems (see, for instance, [20-27] and the references therein). The core idea of the first integral method, through the division theorem for two variables in the complex domain, is to establish a polynomial first integral (with polynomial coefficients) of an explicit form to an autonomous planar system which is equivalent to the equation considered. Taking the derived first integral into account, the method may provide a class of traveling wave solutions in a straightforward manner. In addition, it transforms the problem into a simple algebraic computation.

On the other hand, extending some innovative methods to NEEs for traveling wave solutions seems interesting and important research problem. Hence, we find that substantial work still has to be done in order for the first integral method be well established because every nonlinear equation has its own physically distinct and significant rich structure deserving to be explored further.

The aim of the present paper is to introduce new applications of the first integral method by focusing our attention on two special types of the Schrödinger equations which are of great interest in plasma physics, wave propagation in nonlinear optical fibers, etc. To this end, we organize our paper as follows: in the next section, we describe the method in brief. In Sects. 3 and 4, we analyze our equations for traveling wave solutions. Finally, we state a conclusion in Sect. 5 .

\section{The first integral method}

Suppose that we are given a partial differential equation for a function $u(x, t)$ in the form

$$
P\left(u, u_{t}, u_{x}, u_{t t}, u_{t x}, u_{x x}, \ldots\right)=0,
$$

where $P$ is a polynomial in its arguments while subscripts denote partial derivatives. Through the wave transformation $u(x, t)=U(\xi), \xi=k x-w t+\xi_{0}$, where $k, w$, and 
$\xi_{0}$ are arbitrary constants, Eq. (1) can be converted into an ordinary differential equation (ODE) of the form

$$
P\left(U,-w U^{\prime}, k U^{\prime}, w^{2} U^{\prime \prime},-k w U^{\prime \prime}, k^{2} U^{\prime \prime}, \ldots\right)=0,
$$

where $U=U(\xi)$ and the primes denote ordinary derivatives with respect to $\xi$. Introducing the new variables

$$
X(\xi)=U(\xi), \quad Y(\xi)=\frac{\mathrm{d} U(\xi)}{\mathrm{d} \xi},
$$

we assume that Eq. (2) can be reduced to a planar autonomous system of the form

$$
\begin{aligned}
& \frac{\mathrm{d} X(\xi)}{\mathrm{d} \xi}=Y(\xi), \\
& \frac{\mathrm{d} Y(\xi)}{\mathrm{d} \xi}=Q(X(\xi), Y(\xi)) .
\end{aligned}
$$

Solving a planar autonomous system of ODEs such as (4) directly is a challenging and difficult task in general. Thus, relying on the qualitative theory of ODEs [28], if one can derive a single first integral for the system (4), then one may be able to reduce Eq. (2) to a first-order integrable ODE. Accordingly, a class of traveling wave solutions can be obtained by solving this first-order ODE. However, there is no a systematic approach which can provide us a way of finding the first integrals of Eq. (4), nor is there a logical way for telling us what these first integrals are. On the other hand, looking for the first integrals of ODEs is one of the most important problems because they permit us to get the general solution of a nonlinear differential equation in the form of quadratures. The division theorem for two variables in the complex domain $\mathbb{C}[18,29,30]$ is stated as follows:

Division theorem. Suppose that $P(w, z)$ and $Q(w, z)$ are polynomials in $\mathbb{C}[w, z]$ and $P(w, z)$ is irreducible in $\mathbb{C}[w, z]$. If $Q(w, z)$ vanishes at all zero points of $P(w, z)$, then there exists a polynomial $G(w, z)$ in $\mathbb{C}[w, z]$ such that $Q(w, z)=P(w, z) G(w, z)$.

Remark 1: We note that the division theorem is given in the complex domain $\mathbb{C}$. The real field $\mathbb{R}$ is a subfield of the complex field $\mathbb{C}$, and every algebraic curve is naturally non-degenerate in $\mathbb{C}$. For a given equation in $\mathbb{R}$ we can always extend it to $\mathbb{C}$. If the extended equation has an algebraic curve solution in $\mathbb{C}$, then the intersection of the manifold of this solution and the real plane must be the algebraic curve solution of the original equation in $\mathbb{R}$. If the extended equation has no an algebraic curve solution in $\mathbb{C}$, then the original equation has no algebraic curve solution in $\mathbb{R}$ either. Hence, for a given equation in $\mathbb{R}$, we can use the division theorem in $\mathbb{R}[18]$.

\section{The generalized nonlinear Schrödinger equation}

First, let us consider the generalized nonlinear Schrödinger equation [31] which reads

$$
\begin{aligned}
& u_{t}-\mathrm{i} u_{x x}-\gamma_{1} u_{x}-\gamma_{2}\left(|u|^{2} u\right)_{x}-\gamma_{3}\left(|u|^{2}\right)_{x} u \\
& \quad-\mathrm{i}\left(\gamma_{4}|u|^{2}+\gamma_{5}|u|^{4}\right) u=0,
\end{aligned}
$$

where $\mathrm{i}$ is the imaginary unit, while $\gamma_{j}$ 's are arbitrary real numbers. Equation (5) appears in quantum mechanics. Now, we assume that Eq. (5) admits a solution of the form

$$
\begin{aligned}
& u(x, t)=\varphi(\xi) \exp (\mathrm{i}(\psi(\xi)-\theta t)), \\
& \xi=k x-w t+\xi_{0},
\end{aligned}
$$

where $\varphi$ and $\psi$ are undetermined real functions of the single variable $\xi$, while $\theta, k$, and $w$ are arbitrary constants to be specified, and $\xi_{0}$ is an arbitrary phase shift. Then, setting

$$
\begin{aligned}
& \psi^{\prime}(\xi)=\frac{w+k \gamma_{1}}{2 k^{2}}+\frac{3 \gamma_{2}+2 \gamma_{3}}{4 k} \varphi^{2}(\xi), \\
& \xi=k x-w t+\xi_{0},
\end{aligned}
$$

and substituting (6) into Eq. (5) yield

$$
\begin{aligned}
& \varphi^{\prime \prime}(\xi)+\frac{w^{2}+4 k^{2} \theta+k \gamma_{1}\left(2 w+k \gamma_{1}\right)}{4 k^{4}} \varphi(\xi) \\
& +\frac{\left(w+k \gamma_{1}\right) \gamma_{2}+2 k \gamma_{4}}{2 k^{3}} \varphi^{3}(\xi) \\
& +\frac{\left(\gamma_{2}-2 \gamma_{3}\right)\left(3 \gamma_{2}+2 \gamma_{3}\right)+16 \gamma_{5}}{16 k^{2}} \varphi^{5}(\xi)=0, \\
& \xi=k x-w t+\xi_{0},
\end{aligned}
$$

where the primes denote derivatives with respect to $\xi$. Letting $z=\varphi$ and $y=\varphi^{\prime}$, Eq. (8) can be written as the plane autonomous system

$$
\left\{\begin{array}{l}
\frac{\mathrm{d} z}{\mathrm{~d} \xi}=y \\
\frac{\mathrm{d} y}{\mathrm{~d} \xi}=a z+b z^{3}+c z^{5},
\end{array}\right.
$$

where

$$
\begin{aligned}
& a=-\frac{w^{2}+4 k^{2} \theta+k \gamma_{1}\left(2 w+k \gamma_{1}\right)}{4 k^{4}}, \\
& b=-\frac{\left(w+k \gamma_{1}\right) \gamma_{2}+2 k \gamma_{4}}{2 k^{3}}, \\
& c=-\frac{\left(\gamma_{2}-2 \gamma_{3}\right)\left(3 \gamma_{2}+2 \gamma_{3}\right)+16 \gamma_{5}}{16 k^{2}} .
\end{aligned}
$$

Now, suppose that $z=z(\xi)$ and $y=y(\xi)$ are nontrivial solutions of (9). Also, assume that $q(z, y)=$ $\sum_{i=0}^{m} A_{i}(z) y^{i}$ is an irreducible polynomial in the complex domain $\mathbb{C}$ such that

$$
q(z(\xi), y(\xi))=\sum_{i=0}^{m} A_{i}(z) y^{i}=0
$$

where the polynomials $A_{i}(z)(i=0,1, \ldots, m)$ are relatively prime in $\mathbb{C}$ with $A_{m}(z) \equiv 0$. Equation (10) is called a first integral of Eq. (9). We note that $\mathrm{d} q / \mathrm{d} \xi$ is a polynomial in $z$ and $y$. Thus, $q(z(\xi), y(\xi))=0$ implies that $\mathrm{d} q / \mathrm{d} \xi=0$. Then, by the division theorem, there exists a polynomial $B(z)+C(z) y$ in the complex domain $\mathbb{C}$ such that

$$
\begin{aligned}
\frac{\mathrm{d} q}{\mathrm{~d} \xi} & =\frac{\partial q}{\partial z} \frac{\mathrm{d} z}{\mathrm{~d} \xi}+\frac{\partial q}{\partial y} \frac{\mathrm{d} y}{\mathrm{~d} \xi} \\
& =[B(z)+C(z) y]\left[\sum_{i=0}^{m} A_{i}(z) y^{i}\right] .
\end{aligned}
$$

We consider the case $m=2$ of (10). Hence, taking Eqs. (9) and (11) into account, we get 


$$
\begin{aligned}
& \sum_{i=0}^{2}\left[A_{i}^{\prime}(z) y^{i+1}\right]+\sum_{i=0}^{2}\left[i A_{i}(z) y^{i-1}\left(a z+b z^{3}+c z^{5}\right)\right] \\
& \quad=[B(z)+C(z) y]\left[\sum_{i=0}^{2} A_{i}(z) y^{i}\right] .
\end{aligned}
$$

Equating the coefficients of $y^{i}(0 \leq i \leq 3)$ in Eq. (12) leads to the system

$$
\begin{aligned}
& y^{3}: A_{2}^{\prime}(z)=C(z) A_{2}(z) \\
& y^{2}: A_{1}^{\prime}(z)=B(z) A_{2}(z)+C(z) A_{1}(z) \\
& y^{1}: A_{0}^{\prime}(z)=B(z) A_{1}(z)-2\left(a z+b z^{3}+c z^{5}\right) A_{2}(z) \\
& \quad+C(z) A_{0}(z) \\
& y^{0}: B(z) A_{0}(z)=\left(a z+b z^{3}+c z^{5}\right) A_{1}(z) .
\end{aligned}
$$

From Eq. (13), we obtain $A_{2}(z)=c_{0} \exp \left(\int C(z) \mathrm{d} z\right)$, where $c_{0}$ is an integration constant. Since $A_{2}(z)$ and $C(z)$ are polynomials, we deduce that $C(z)=0$ and $A_{2}(z)$ must be a constant. For simplicity, we can take $A_{2}(z)=1$. Then, Eqs. (14) and (15) reduce to the following equations:

$$
\begin{aligned}
& A_{1}^{\prime}(z)=B(z), \\
& A_{0}^{\prime}(z)=B(z) A_{1}(z)-2\left(a z+b z^{3}+c z^{5}\right) .
\end{aligned}
$$

At this stage, it becomes obligatory to make the following case analysis:

Case 1. $\operatorname{deg} A_{1}(z)=0$.

In this case, from (16) and (17), we conclude that $A_{1}(z) \equiv 0$. Then, (18) gives

$$
A_{0}(z)=-\frac{c}{3} z^{6}-\frac{b}{2} z^{4}-a z^{2}
$$

where we set the integration constant to zero for simplicity. Hence, Eq. (10) becomes

$$
-\frac{c}{3} z^{6}-\frac{b}{2} z^{4}-a z^{2}+y^{2}=0
$$

which is a first integral of Eq. (9). Solving Eq. (20) for $y$, we get

$$
y= \pm \sqrt{\frac{c}{3} z^{6}+\frac{b}{2} z^{4}+a z^{2}} .
$$

Here and henceforth, the signs $( \pm)$ or $(\mp)$ are ordered vertically. Combining the first equation of (9) with Eq. (21), solving the resulting equation by a quadrature, and changing to the original variables, we obtain

Observation 1. Equation (8) admits an exact solution of the form

$$
\begin{gathered}
\varphi(\xi)= \pm\left(48 a /\left(\sqrt{6}\left(6 b^{2}-32 a c+1\right) \cosh (2 \sqrt{a} \xi)\right.\right. \\
\left.\left.+\sqrt{6}\left(6 b^{2}-32 a c-1\right) \sinh (2 \sqrt{a} \xi)-12 b\right)\right)^{1 / 2},
\end{gathered}
$$

where $\xi=k x-w t+\xi_{0}, a, b$, and c are as in (9), while all involved constants remain arbitrary; Eq. (5) admits a traveling wave solution of the form

$$
u(x, t)=\varphi(\xi) \exp (\mathrm{i}(\psi(\xi)-\theta t)),
$$

where $\varphi(\xi)$ is described by (22), and $\psi(\xi)$ is defined as in (7).

Case 2. $\operatorname{deg} A_{1}(z)=1$ or $\operatorname{deg} A_{1}(z)=2$.

In this case, we end up with the same first integral as (20). We omit to present the details for brevity.

Case 3. $\operatorname{deg} A_{1}(z)=3$.

In this case, from (16) and (17), we conclude that $\operatorname{deg} B(z)=2$ and $\operatorname{deg} A_{0}(z)=6$. Assuming $A_{1}(z)=$ $a_{3} z^{3}+a_{2} z^{2}+a_{1} z+a_{0}\left(a_{3} \neq 0\right)$ and $B(z)=b_{2} z^{2}+b_{1} z+$ $b_{0}\left(b_{2} \neq 0\right)$ in $(17)$, we get $b_{2}=3 a_{3}, b_{1}=2 a_{2}$, and $b_{0}=a_{1}$. Substituting $A_{1}(z)$ and $B(z)$ into Eq. (18) and integrating the resulting equation leads to

$$
\begin{aligned}
& A_{0}(z)=\frac{3 a_{3}^{2}-2 c}{6} z^{6}+a_{2} a_{3} z^{5}+\frac{a_{2}^{2}+2 a_{1} a_{3}-b}{2} z^{4} \\
& \quad+\left(a_{1} a_{2}+a_{0} a_{3}\right) z^{3}+\frac{a_{1}^{2}+2 a_{0} a_{2}-2 a}{2} z^{2} \\
& \quad+a_{0} a_{1} z+d,
\end{aligned}
$$

where $d$ denotes an integration constant. Then, substituting $A_{0}(z), A_{1}(z)$, and $B(z)$ into Eq. (16), equating the coefficients of $z^{i}(0 \leq i \leq 8)$ to zero, and solving the resulting system of nonlinear algebraic equations simultaneously, we get the relations

$$
\begin{aligned}
& d=0, \quad a_{3}=-2 \sqrt{\frac{c}{3}}, \quad a_{1}=\mp 2 \sqrt{a}, \\
& b= \pm 4 \sqrt{\frac{a c}{3}}, \quad a_{0}=0, \quad a_{2}=0 \\
& d=0, \quad a_{3}=2 \sqrt{\frac{c}{3}}, \quad a_{1}=\mp 2 \sqrt{a}, \\
& b=\mp 4 \sqrt{\frac{a c}{3}}, \quad a_{0}=0, \quad a_{2}=0,
\end{aligned}
$$

where all other constants remain arbitrary. Hence, Eq. (10) becomes

$$
\begin{gathered}
y^{2}+\left(-2 \sqrt{\frac{c}{3}} z^{3} \mp 2 \sqrt{a} z\right) y+a z^{2} \\
\pm 2 \sqrt{\frac{a c}{3}} z^{4}+\frac{c}{3} z^{6}=0, \\
y^{2}+\left(2 \sqrt{\frac{c}{3}} z^{3} \mp 2 \sqrt{a} z\right) y+a z^{2} \\
\mp 2 \sqrt{\frac{a c}{3}} z^{4}+\frac{c}{3} z^{6}=0,
\end{gathered}
$$

which are first integrals of Eq. (9). Solving Eqs. (26) and (27) for $y$, respectively, we get

$$
\begin{aligned}
& y=\sqrt{\frac{c}{3}} z^{3} \pm \sqrt{a} z, \\
& y=-\sqrt{\frac{c}{3}} z^{3} \pm \sqrt{a} z .
\end{aligned}
$$

Combining the first equation of (9) with Eqs. (28) and (29), solving the resulting equations by quadratures, and changing to the original variables, we obtain

Observation 2. Equation (8) admits exact solutions of the form

$$
\varphi(\xi)=\mp\left(\frac{3 \sqrt{a}}{\cosh (2 \sqrt{a} \xi)-\sinh (2 \sqrt{a} \xi)-\sqrt{3 c}}\right)^{1 / 2}
$$




$$
\varphi(\xi)=\mp\left(\frac{3 \sqrt{a}}{\cosh (2 \sqrt{a} \xi)+\sinh (2 \sqrt{a} \xi)-\sqrt{3 c}}\right)^{1 / 2}
$$

where $\xi=k x-w t+\xi_{0}, a, b$, and $c$ are as in (9) with $3 b^{2}=16 a c$, while all involved constants remain arbitrary; $E q$. (5) admits a traveling wave solution of the form

$$
u(x, t)=\varphi(\xi) \exp (\mathrm{i}(\psi(\xi)-\theta t)),
$$

where $\varphi(\xi)$ is described by (30) or (31), and $\psi(\xi)$ is defined as in (7).

Remark 2. If $\operatorname{deg} A_{1}(z)=k>3$, then we deduce that $\operatorname{deg} B(z)=k-1$ and $\operatorname{deg} A_{0}(z)=2 k$ due to Eqs. (17) and (18). However, this yields a contradiction with Eq. (16) because the degree of the polynomial $B(z) A_{0}(z)$ is $3 k-1$ while the degree of the polynomial $\left(a z+b z^{3}+c z^{5}\right) A_{1}(z)$ is $k+5$.

Remark 3. It is worth to mention here that, in [32], a bounded traveling wave solution was proposed to the two-dimensional Korteweg-de Vries-Burgers equation by analysing some cases similar to Eq. (8). In addition, a special case of Eq. (5) was also solved by a similar ansatz [33].

\section{The Schrödinger equation with Kerr law nonlinearity}

Next, we consider the higher-order nonlinear Schrödinger equation with the Kerr law nonlinearity in the form

$$
\begin{aligned}
u_{t} & =\mathrm{i} \gamma_{1} u_{x x}+\mathrm{i} \gamma_{2} u|u|^{2}+\gamma_{3} u_{x x x}+\gamma_{4}\left(u|u|^{2}\right)_{x} \\
& +\gamma_{5} u\left(|u|^{2}\right)_{x}
\end{aligned}
$$

which describes propagation of pulses [34], where $u=u(x, t)$ is a complex function, $\mathrm{i}$ is the imaginary unit, and $\gamma_{j}(0 \leq j \leq 5)$ are arbitrary real parameters. In fact, Eq. (32) is a simple Sasa-Satsuma equation that arises in nonlinear fiber optics (predominantly). In order to solve Eq. (32), we take the gauge transformation

$$
\begin{aligned}
& u(x, t)=\psi(\xi) \exp (\mathrm{i}(\alpha x-\beta t)), \\
& \xi=k x-w t+\xi_{0}
\end{aligned}
$$

into account, where $\psi=\psi(x, t)$ is a real function, $\alpha$, $\beta, k$, and $w$ are arbitrary constants to be specified, $\xi_{0}$ is an arbitrary phase shift. Substituting (33) into Eq. (32), and equating the real and imaginary parts to zero, respectively, we get the real system of ordinary differential equations

$$
\begin{aligned}
& k^{2}\left(\gamma_{1}+3 \alpha \gamma_{3}\right) \psi^{\prime \prime}+\left(\beta-\alpha^{2} \gamma_{1}-\alpha^{3} \gamma_{3}\right) \psi \\
& \quad+\left(\gamma_{2}+\alpha \gamma_{4}\right) \psi^{3}=0, \\
& k^{3} \gamma_{3} \psi^{\prime \prime \prime}+\left(w-2 k \alpha \gamma_{1}-3 k \alpha^{2} \gamma_{3}\right) \psi^{\prime} \\
& \quad+\left(3 k \gamma_{4}+2 k \gamma_{5}\right) \psi^{2} \psi^{\prime}=0,
\end{aligned}
$$

where $\psi=\psi(x, t)$ and the primes denote derivatives with respect to $\xi$. Under the constraint conditions

$$
\begin{aligned}
& \alpha=\frac{3 \gamma_{2} \gamma_{3}-\gamma_{1}\left(3 \gamma_{4}+2 \gamma_{5}\right)}{6 \gamma_{3}\left(\gamma_{4}+\gamma_{5}\right)}, \\
& \beta=\frac{\gamma_{1}\left(w-2 k \alpha \gamma_{1}\right)+\alpha\left(3 w-8 k \alpha \gamma_{1}\right) \gamma_{3}-8 k \alpha^{3} \gamma_{3}^{2}}{k \gamma_{3}},
\end{aligned}
$$

Eqs. (34) and (35) turn out to be

$$
\psi^{\prime \prime}-\frac{2 k \alpha \gamma_{1}+3 k \alpha^{2} \gamma_{3}-w}{k^{3} \gamma_{3}} \psi+\frac{3 \gamma_{4}+2 \gamma_{5}}{3 k^{2} \gamma_{3}} \psi^{3}=0 .
$$

Setting $z=\psi$ and $y=\psi^{\prime}$ in Eq. (37), we obtain the equivalent planar system

$$
\left\{\begin{array}{l}
\frac{\mathrm{d} z}{\mathrm{~d} \xi}=y, \\
\frac{\mathrm{d} y}{\mathrm{~d} \xi}=\frac{2 k \alpha \gamma_{1}+3 k \alpha^{2} \gamma_{3}-w}{k^{3} \gamma_{3}} z-\frac{3 \gamma_{4}+2 \gamma_{5}}{3 k^{2} \gamma_{3}} z^{3} .
\end{array}\right.
$$

From now on, we shall omit some of the details because the procedure is the same. According to the first integral method, we consider the case $m=2$ of (10). Then, by equating the coefficients of $y^{i}(0 \leq i \leq 3)$ on both sides of (11), we have

$$
\begin{aligned}
& y^{3}: A_{2}^{\prime}(z)=C(z) A_{2}(z), \\
& y^{2}: A_{1}^{\prime}(z)=C(z) A_{1}(z)+B(z) A_{2}(z), \\
& y^{1}: A_{0}^{\prime}(z)=C(z) A_{0}(z)+B(z) A_{1}(z) \\
& \quad-2\left(\frac{2 k \alpha \gamma_{1}+3 k \alpha^{2} \gamma_{3}-w}{k^{3} \gamma_{3}} z-\frac{3 \gamma_{4}+2 \gamma_{5}}{3 k^{2} \gamma_{3}} z^{3}\right) \\
& \quad \times A_{2}(z), \\
& y^{0}: B(z) A_{0}(z)=A_{1}(z)\left(\frac{2 k \alpha \gamma_{1}+3 k \alpha^{2} \gamma_{3}-w}{k^{3} \gamma_{3}} z\right. \\
& \left.\quad-\frac{3 \gamma_{4}+2 \gamma_{5}}{3 k^{2} \gamma_{3}} z^{3}\right) .
\end{aligned}
$$

Since $A_{2}(z)$ and $C(z)$ are polynomials, from Eq. (39) we deduce that $C(z)=0$ and $A_{2}(z)$ must be a constant. For simplicity, we can take $A_{2}(z)=1$. Balancing the degrees of $A_{0}(z), A_{1}(z)$, and $B(z)$, we conclude that $\operatorname{deg} B(z)=1$ only. Suppose that $B(z)=b_{1} z+b_{0}\left(b_{1} \neq 0\right)$. Then, from (40) and (41), we get

$$
\begin{aligned}
& A_{1}(z)=\frac{b_{1}}{2} z^{2}+b_{0} z+e, \\
& \begin{array}{l}
A_{0}(z)=\left(\frac{b_{1}^{2}}{8}+\frac{3 \gamma_{4}+2 \gamma_{5}}{6 k^{2} \gamma_{3}}\right) z^{4}+\frac{b_{1} b_{0}}{2} z^{3} \\
\quad+\left(\frac{e b_{1}+b_{0}^{2}}{2}-\frac{2 k \alpha \gamma_{1}+3 k \alpha^{2} \gamma_{3}-w}{k^{3} \gamma_{3}}\right) z^{2} \\
\quad+e b_{0} z+f,
\end{array}
\end{aligned}
$$

where $e$ and $f$ are integration constants. Substituting $A_{0}(z), A_{1}(z)$, and $B(z)$ into (42) and setting the coefficients of $z^{i}(0 \leq i \leq 5)$ to zero, we derive a system of nonlinear algebraic equations for $b_{0}, b_{1}, e, f, k, w, \alpha$, and $\gamma_{i}$ 's. Solving the resultant system simultaneously, we get the solution set

$$
\begin{aligned}
& f=-\frac{3\left(w-2 k \alpha \gamma_{1}-3 k \alpha^{2} \gamma_{3}\right)^{2}}{2 k^{4} \gamma_{3}\left(3 \gamma_{4}+2 \gamma_{5}\right)}, \\
& e=\mp \frac{\sqrt{6}\left(k \alpha\left(2 \gamma_{1}+3 \alpha \gamma_{3}\right)-w\right)}{k^{2} \sqrt{-\gamma_{3}\left(3 \gamma_{4}+2 \gamma_{5}\right)}}, \\
& b_{0}=0, b_{1}= \pm \frac{2}{k} \sqrt{-\frac{6 \gamma_{4}+4 \gamma_{5}}{3 \gamma_{3}}},
\end{aligned}
$$

where all other constants remain arbitrary. Using the relation (45) in (10), we get 


$$
\begin{aligned}
y= & \mp\left(\frac{2 k \alpha \gamma_{1}+3 k \alpha^{2} \gamma_{3}-w}{k^{3} \gamma_{3}}-\frac{3 \gamma_{4}+2 \gamma_{5}}{3 k^{2} \gamma_{3}} z^{2}\right) \\
& / \frac{1}{k} \sqrt{-\frac{6 \gamma_{4}+4 \gamma_{5}}{3 \gamma_{3}}} .
\end{aligned}
$$

Combining the first equation of (38) with Eq. (46), solving the resulting equations by quadratures, and changing to the original variables, we obtain

Observation 3. Equation (32), under the condition (36), admits exact solutions of the form

$$
\begin{aligned}
& u_{1}^{ \pm}(x, t)= \pm \sqrt{\frac{3\left(w-2 k \alpha \gamma_{1}-3 k \alpha^{2} \gamma_{3}\right)}{3 k \gamma_{4}+2 k \gamma_{5}}} \\
& \quad \times \tan \left(\sqrt{\frac{2 k \alpha \gamma_{1}+3 k \alpha^{2} \gamma_{3}-w}{2 k^{3} \gamma_{3}}}\left(k x-w t+\xi_{0}\right)\right) \\
& \quad \times \exp (\mathrm{i}(\alpha x-\beta t)),
\end{aligned}
$$

where $\frac{w-2 k \alpha \gamma_{1}-3 k \alpha^{2} \gamma_{3}}{k^{3} \gamma_{3}}<0$ and $\frac{3 \gamma_{4}+2 \gamma_{5}}{k^{2} \gamma_{3}}<0$, while all involved constants remain arbitrary; and

$$
\begin{aligned}
& u_{2}^{ \pm}(x, t)= \pm \sqrt{\frac{3\left(2 k \alpha \gamma_{1}+3 k \alpha^{2} \gamma_{3}-w\right)}{3 k \gamma_{4}+2 k \gamma_{5}}} \\
& \quad \times \tanh \left(\sqrt{\frac{w-2 k \alpha \gamma_{1}-3 k \alpha^{2} \gamma_{3}}{2 k^{3} \gamma_{3}}}\left(k x-w t+\xi_{0}\right)\right) \\
& \quad \times \exp (\mathrm{i}(\alpha x-\beta t)), \\
& \text { where } \frac{w-2 k \alpha \gamma_{1}-3 k \alpha^{2} \gamma_{3}}{k^{3} \gamma_{3}}>0 \text { and } \frac{3 \gamma_{4}+2 \gamma_{5}}{k^{2} \gamma_{3}}<0 \text {, while all } \\
& \text { involved constants remain arbitrary. }
\end{aligned}
$$

\section{Conclusion}

Exact and explicit solutions when they are available can help us to well understand the mechanism of the complicated physical phenomena modeled by NEEs. First integrals play a crucial role in the study of nonlinear ordinary differential equations because they permit one to solve a nonlinear differential equation by quadratures. In this study, we observed that the first integral method can be a useful tool for discovering first integrals of higher-order nonlinear Schrödinger-type equations by analyzing two specific equations. The method seems to be applicable to other physically important NEEs which can be converted to a second-order ordinary differential equation through a traveling wave transformation.

\section{Acknowledgments}

The author is grateful to the referee for the recommendation of some useful references.

\section{References}

[1] J. Weiss, M. Tabor, G. Carnevale, J. Math. Phys. 24, $522(1983)$

[2] M.J. Ablowitz, H. Segur, Solitons and Inverse Scattering Transform, SIAM, Philadelphia 1981.
[3] A.M. Wazwaz, Appl. Math. Comput. 201, 489 (2008)

[4] W.X. Ma, Chaos Solitons Fract. 42, 1356 (2009)

[5] G.W. Bluman, S. Kumei, Symmetries and Differential Equations, Springer-Verlag, New York 1989.

[6] A.M. Wazwaz, Appl. Math. Comput. 154, 713 (2004)

[7] M.L. Wang, Phys. Lett. A 213, 279 (1996)

[8] M.A. Abdou, Chaos Solitons Fract. 31, 95 (2007)

[9] M. Wang, X. Li, J. Zhang, Phys. Lett. A 372, 417 (2008).

[10] J.H. He, X.H. Wu, Chaos Solitons Fract. 30, 700 (2006)

[11] J.H. He, Appl. Math. Comput. 151, 287 (2004)

[12] A. Biswas, H. Triki, Appl. Math. Comput. 217, 3869 (2010)

[13] D. Wang, H.Q. Zhang, Chaos Solitons Fract. 25, 601 (2005)

[14] W.X. Ma, T. Huang, Y. Zhang, Phys. Scr. 82, 065003 (2010)

[15] M. Dehghan, A. Hamidi, M. Shakourifar, Appl. Math. Comput. 189, 1034 (2007)

[16] W.X. Ma, Y. Zhang, Y. Tang, J. Tu, Appl. Math. Comput. 218, 7174 (2012)

[17] Z. Feng, Phys. Lett. A. 293, 57 (2002)

[18] Z. Feng, X. Wang, Phys. Scr. 64, 7 (2001)

[19] Z. Feng, J. Phys. A, Math. Gen. 35, 343 (2002)

[20] X. Deng, Appl. Math. Comput. 204, 733 (2008).

[21] Z. Feng, S. Zheng, D.Y. Gao, Z. Angew. Math. Phys. 60, 756 (2009).

[22] Naranmandula, K.X. Wang, Chin. Phys. 13, 139 (2004).

[23] Z. Feng, R. Knobel, J. Math. Anal. Appl. 328, 1435 (2007)

[24] İ. Aslan, Appl. Math. Comput. 217, 8134 (2011)

[25] S. Abbasbandy, A. Shirzadi, Commun. Nonlinear Sci. Numer Simul. 15, 1759 (2010)

[26] K.R. Raslan, Nonlinear Dyn. 53, 281 (2006)

[27] İ. Aslan, Pramana-J. Phys. 76, 1 (2011).

[28] T.R. Ding, C.Z. Li, Ordinary Differential Equations, Peking University Press, Peking 1996.

[29] Z. Feng, Electron J. Linear Algebra 8, 14 (2001).

[30] N. Bourbaki, Commutative Algebra, Addison-Wesley, Paris 1972.

[31] C.Q. Bian, J. Pang, L.H Jin, X.M. Ying, Commun. Nonlinear Sci. Numer. Simul. 15, 2337 (2010)

[32] W.X. Ma, J. Phys. A, Math. Gen. 26, L17 (1993)

[33] W.X. Ma, M. Chen, Appl. Math. Comput. 215, 2835 (2009)

[34] A. Biswas, S. Konar, Introduction to Non-Kerr Law Optical Solitons, CRC Press, Florida 2007. 